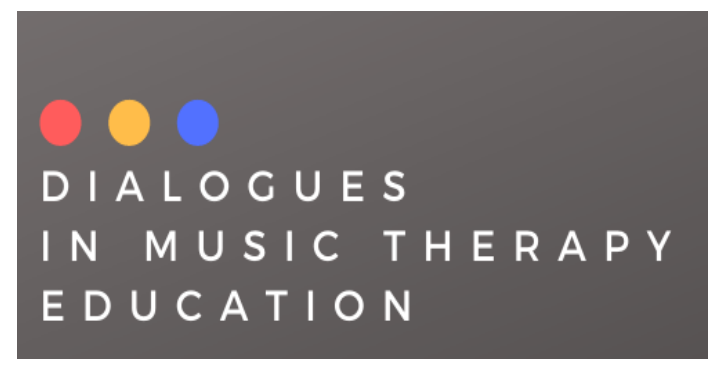

\title{
EDITORIAL
}

\section{Do we need another journal?}

Meganne K. Masko, PhD, MT-BC/L

Editor

Carolyn Moore, $\mathrm{PhD}$, MT-BC

Associate Editor for Communication

Natasha Thomas, PhD, MT-BC

Associate Editor for Creative/Arts-Based Submissions

Virginia Driscoll, PhD, MT-BC

Associate Editor for Book Reviews 
Do We Need Another Journal?

I (Meganne Masko) was sitting at my dining room table working on an article draft in the summer of 2019 when I texted Dr. Carolyn Moore (Sam Houston State University) and asked what she thought about starting an open access music therapy journal dedicated to education and clinical training. To my great delight, she said it sounded like a good idea. We researched opportunities for open access journals at our respective institutions and ultimately decided that IUPUI would host the new publication. We then set about the process of bringing our idea to life.

\section{In What Ways Is Dialogues Different?}

The term dialogue implies a conversation, an exchange of ideas, a dynamic interaction that leads to deeper understanding, yields new insights, or improves relationships. In formal educational spaces, dialogue may be stifled for a variety of reasons, some of which may boil down to a simple challenge of focus and clarity.

The nature of language is that it is constantly evolving. The terms we use and the framing we provide around them can mean the difference between clarity of intent that can lead to meaningful results, or an obstacle course of confusion that can stall or altogether halt even the most promising of projects. Researchers know that sometimes a narrow focus can be an asset; it's why we lean into our specializations. An intentionally narrow focus can provide necessary opportunities for depth and exploration into topics (and sub-topics!) that there may not be space for in broader discussion, while ultimately serving to strengthen the foundations for all the places where knowledge is generated, when we honor the time and space it takes to dive deep. And so, we arrive at the purpose of this journal.

One of the many items on the journal's "to do" list was to assemble an editorial board. Drs. Virginia Driscoll and Natasha Thomas joined as associate editors. 
Our work then became to build a frame around the intentionally narrow topic of music therapy education and clinical training that was still accessible to a broader community of readers, in order to provide the kind of meaningful and equitable generative space that we believe could best benefit the profession of music therapy.

We specifically selected a platinum open access journal format because, in our view, it best fit the idea of a journal being an opportunity to encourage dialogue with and between as many stakeholders involved in music therapy education and clinical training as possible. Platinum open access journals such as Dialogues are unique because there are no fees associated with publishing or accessing any journal content, thus removing the financial barriers often in place in other journals, including those that publish in other open access formats. Financial barriers associated with journals mean that only authors and researchers with access to funds to publish or access the publication are able to do so, closing the door for dialogues that meaningfully include student, supervisor, junior faculty, and other diverse perspectives. We thank Indiana University for its investment in open access materials.

In addition to the platinum open access format, we decided to intentionally engage broadly within the narrow focus of music therapy education by embracing submissions representative of a wide range of research methodologies and submission formats. In doing so, we hope to engage in dialogues that do not center a particular tradition or group of stakeholders, but embrace multiple ways of knowing, sharing, and understanding.

Ultimately, our goal here is to engage the music therapy community in creative and critical conversations about all aspects of teaching and learning. 